\title{
Hyperkinetic Syndrome in Adolescent with HIV-Infection
}

\author{
Borysova TP, Badogina LP*, Allahverdieva ZS and Samsonenko SV \\ SI “Dnepropetrovsk Medical Academy, Ministry of Health of Ukraine”, Dnipro, Ukraine \\ *Corresponding Author: Badogina LP, SI "Dnepropetrovsk Medical Academy, \\ Ministry of Health of Ukraine”, Dnipro, Ukraine.
}

Received: February 27, 2020

Published: April 23, 2020

(C) All rights are reserved by Badogina LP.,

et al.

\section{Abstract}

A clinical case of a rare manifestation of HIV encephalopathy in a teenager in the form of generalized hyperkinesis is described. Differential diagnosis with acute rheumatic fever, systemic connective tissue disease, demyelinating diseases, tumors of the central nervous system, Wilson-Konovalov disease is presented.

Keywords: HIV Infection; HIV Encephalopathy; Chorea; Children

\section{Introduction}

The disease caused by the human immunodeficiency virus (HIV) or HIV infection - slowly progressive antroponic disease that is characterized by damage of the immune system with the development of acquired immunodeficiency syndrome (AIDS) - the final stage of HIV infection, accompanied by primary or secondary lesion of all organs and systems.

The HIV epidemic remains a serious problem worldwide. According to WHO statistics, 37.9 million people including 1.7 million children are living with HIV today [1]. Ukraine takes one of the first places in the European region for the number of HIV-infected people. In Dnipropetrovsk region, which traditionally has a leading position in the level of HIV prevalence, the largest number of children aged 0 to 18 years who are registered for the HIV - 599, including 166 in the stage of AIDS. Late diagnosis of HIV infection in the Dnipropetrovsk region keeps rather high level and remains virtually unchanged. The share of people with newly diagnosed at stage IIIIV disease was in 2013 - 58.0\% 2016 - 57.5\% 2018 - 54.9\% [2].

The earliest manifestations of HIV infection is increasing lymph nodes of two different groups and more, hepatolienal syndrome, recurrent bacterial infections, namely: purulent otitis media, meningitis, skin, bones or joints lesions, pneumonia with signs of destruction, repeated episodes of bacterial sepsis, diarrhea progressive weight loss. Opportunistic disease - cytomegalovirus infection, cryptococcosis, candidiasis, tuberculosis, pneumocystis pneumonia, toxoplasmosis, etc. - have malignant course and are major causes of mortality. One of constant syndromes in children with HIV is also central nervous system (CNS) injury. According to the literature, $70 \%$ of HIV-infected adults and $90 \%$ of children have different neurological symptoms that develop at all stages of the disease [3-6].

Neurologic manifestations of the disease may occur due to the direct effect of HIV on the nervous system, opportunistic infections and malignant tumors arising because of immunosuppression, neurotoxic effects of antiretroviral therapy and other systemic complications of HIV that affect brain function [7-9]. Primary infection occurs in cells of the CNS microglia due to infected macrophages penetration through the blood-brain barrier, and gaps between endothelial cells of capillaries. Over time this leads to dysfunction of neurons, astrocytes, microglia, their death due to toxic effects on neurons virus proteins and cytokines [10]. It is known that HIV selectively affects the cells that carry the membrane antigen CD4. Neurons haven't receptor CD4, required for HIV infection, and HIV is not detected inside of patients' neurons with HIV-associated CNS disease. However neuronal receptor expression profile, which comprises jointly receptor HIV CCR5 and CXCR4, which makes them susceptible to damage with viral proteins and inflammatory mediators caused by HIV infection [10].

The impact of HIV infection on the relatively immature nervous system of children infected with HIV in the perinatal period is significant, but the pathogenesis of delayed nervous system damage has not yet been fully understood. The central nervous system is exposed to the virus in the early stages of systemic HIV infection, accompanied by an immune response within the brain. However, clinically significant lesions of the nervous system in the most patients often occur on the background of profound immunosuppression and in the presence of other signs of HIV infection. 
HIV-associated neurological disorders are very variable. Primary lesions may be represented by HIV-associated neurocognitive impairment, encephalopathy (AIDS-dementia), myelopathy, polyneuropathy, aseptic meningitis (meningoencephalitis). In 45\% of patients with HIV neurological symptoms appear at the onset of the disease [11-13]. They may be manifested in the central and/or peripheral nervous system; have an acute, subacute, or slow progressive course.

Early penetration of the virus into the CNS, affecting the brain of the fetus and baby, is thought to lead to the most common primary CNS complication associated with human immunodeficiency virus - HIV-encephalopathy [14-16]. Clinical symptoms may vary and depend on the child age and the method of HIV infection. Infants and young children exhibit the most severe and global neurological dysfunction, while older children have more specific signs. The severity of the disease and the infant's early age are considered unfavorable prognostic factors for neurocognitive functioning.

HIV-encephalopathy in children may have a progressive (subacute and plateau types) and static options [17]. Subacute type of progressive encephalopathy is characterized by loss of previously acquired language and motor skills, progressive movement disorders, acquired microcephaly, pyramidal insufficiency, cerebellar disorders and other. In the "plateau" type acquired skills are not lost, but the further development of the child becomes much slower. In the static version, the acquired skills are not lost, the further development of the child continues, but the children acquire new skills and abilities more slowly than usual. Motor disorders are without signs of progression [18].

The symptoms of HIV-encephalopathy in school-aged children may be manifested as deficiencies in attention span and executive behavior, which means an inability to direct their actions toward active problem solving. Such children can often be distracted and more impulsive, have difficulties in planning and organizing, and be incapable of solving problems [8]. Deficiencies in visual-spatial processing, visual-motor integration and fine motor skills have also been demonstrated in HIV-infected children and may manifest as learning disabilities, poor handwriting, and problems associated with performing certain activities in daily life (e.g. dressing) [8]. One of the manifestations that occurs during the growth and development of a child with HIV infection is the acquired symmetric motor deficit accompanied by paresis, pathological reflexes, ataxia and/or gait disorders [8]. Impairment of motor development occurs in $67 \%$ of HIV- infected children [12,19].

In order to confirm HIV-associated encephalopathy, in addition to general clinical tests conducted for the diagnosis of HIV, neuroimaging is required: computed tomography (CT) and magnetic resonance imaging (MRI). These studies are indicated for all HIV-pos- itive children with altered mental status, headache, convulsions, or focal neurological signs [20]. CT scan does not detect any changes in brain or may be mild atrophy with areas of low density in the white matter. According to MRI, the most common brain lesion is diffuse atrophy, which is observed in $31 \%$ of patients with asymptomatic HIV infection and in $70 \%$ of patients with clinical manifestations of AIDS, signs of leukoencephalopathy. These changes never apply to areas of the cerebral cortex. Sometimes in addition to atrophy of the substance of the brain, there is an dilatation of the ventricles and furrows of the cerebral hemispheres [20].

Unfortunately, HIV infection is rarely considered among the possible etiological factors of the detected neurological pathology. This is one of the reasons for late diagnosis of the disease, which leads to the untimely start of specific therapy, worsens the prognosis and contributes to the spread of infection in the population.

Chorea syndrome is considered as a possible clinical variant of CNS HIV in adult patients [21,22]. In the available literature, we have not encountered reports of the development of choreoid syndrome in HIV-infected children.

The following clinical case demonstrates the diagnosis of latestage HIV infection in a 13-year-old child with chorea syndrome.

\section{Case Report}

Adolescent Y., 13, hospitalized to the Dnepropetrovsk Regional child's clinical hospital in september, 2019 with complaints for the uncoordinated violent motions of extremities. Anamnesis of disease: single hyperkinesias have arisen in April, 2019. At that time there was also an episode of arthritis of the left elbow joint during for the $1^{\text {st }}$ week. Therapy did not use. After 5 months strengthening hyperkinesis were marked. Children's cardiologist residence suggested on rheumatic chorea. A child was directed to cardiac-rheumatology department.

Life history: Patient is the $2^{\text {nd }}$ baby from pregnancy with preeclampsia in the first trimester and the $2^{\text {nd }}$ delivery. He was born in the term of 38 weeks with signs of intrauterine growth retardation, weight - $2510 \mathrm{~g}$, height - $49 \mathrm{~cm}$. Postponed disease (data from ambulatory card): aged 1 month - atopic dermatitis, oral candidiasis, 1 year - cervical lymphadenopathy, 12 years - dysplastic cardiomyopathy, mitral valve prolapse.

Physical examination: The state was severe with marked signs of ballism of the head, upper and lower limbs. No fever. Physical development corresponds to age (weight - $42 \mathrm{~kg}$, height $-160 \mathrm{~cm}$, BMI $-16.0 \mathrm{~kg} / \mathrm{m}^{2}$ ). Cheilitis. Cervical lymphadenopathy. Breathing is vesicular. Heart sounds are sonorous, apical systolic murmur. The liver and spleen are not enlarged. Bowel movements 1 per day. Urination is not violated. 
Neurological status: Patient has clear mind, is focused in the place, time and person. He does not have meningeal signs, asymmetrical face. Taste, hearing are not violated. Swallowing saved. There have been persistent generalized choreoid hyperkinesis in the extremities, rarely of the facial muscles. The movements seem bizarre. Pupils D = S, photoreaction is saved. Cranial nerves are without apparent disease. The tongue is in the midline. Speech is intermittent with mild dysarthria. Tendon reflexes are increased, $\mathrm{S} \geq \mathrm{D}$. Symptom Babinski is fuzzy on both sides. Muscle strength is preserved in the limbs. Muscle tone is satisfactory. Sensitivity violations were not found. Patient is unstable in Romberg's pose, coordination tests performs with discoordination especially in the left side. He walks themselves and has dancing gait on widely set feet.

Preliminary diagnosis: Generalized hyperkinetic syndrome, bilateral cerebellar failure, reflex hemiparesis of the left side.

To clarify the genesis choreic hyperkinesis and holding the differential diagnosis of acute rheumatic fever, systemic connective tissue diseases, demyelinating diseases, body-organic disorders of the central nervous system, Wilson - Konovalov disease examination was assigned: complete blood count, C-reactive protein, antistreptolysin-O (ASLO), echocardiography, ECG, native DNA antibodies, antinuclear antibody levels of copper, ceruloplasmin in the blood, C-reactive protein, brain MRI.

The results of the survey. Complete blood count, HB - $119 \mathrm{~g} / \mathrm{L}$, RBC $-4.23^{12} / \mathrm{L}$, color index -0.84 , HT $-33 \%$, platelets $-1379 / \mathrm{L}$, WBC $-3.6^{9} / \mathrm{L}$, ECR $-16 \mathrm{~mm} /$ hour, band form - 10\%, segmented $70 \%$ eosinophils - $4 \%$, lymphocytes - $8 \%$ monocytes - 7\%.

C-reactive protein $-<6 \mathrm{mg} / \mathrm{l}$, ASLO $-<200 \mathrm{IU} / \mathrm{L}$.

Echocardiography: mitral valve prolapse with regurgitation to the roof of the left atrium.

\section{ECG: sinus arrhythmia.}

Consulting of an ophthalmologist, partial atrophy of the optic nerve in both eyes, mild myopia in the both eyes.

Clinical data and the results of examination allowed to exclude the diagnosis of rheumatic chorea due to absence of muscular hypotonia, signs of carditis, arthritis, rheumatic history, fever, leukocytosis, normal levels of C-reactive protein and ASLO.

Antibodies to native DNA, antinuclear antibody - were not found. Clinical and laboratory evidence of systemic connective tissue diseases, including systemic lupus erythematosus are not.

The normal levels of copper, ceruloplasmin in blood allowed to exclude Wilson-Konovalov disease.
The presence of thrombocytopenia and leukopenia were the basis for the study of myelogram. According to the results myeloproliferative blood disorders was excluded.

MRI of the brain - moderate atrophy of the cerebral hemispheres vicarious ventricle dilatation phenomena. MR data of demyelinating disease, volume organic CNS pathology were not found.

Because of the resistance of neurological disorders, the presence of persistent cervical lymphadenopathy, thrombocytopenia, leukopenia, lymphopenia, after pretest counseling mothers and obtain information consent HIV testing, the child was examined. Result ELISA blood was HIV positive. In the immunological study of blood child very severe immunosuppression was identified - CD4 + T lymphocytes - 39 cells $/ 1$ mkl (4.6\%). PCR viral load - HIV RNA in plasma - 87586 copies $/ \mathrm{ml}$.

Afterwards, after-test counseling was conducted with the mother regarding the HIV detection in her child, also, anamnestic data was completed. The mother appeared to have consumed injected drugs. HIV-status in her was determined in the first half of the pregnancy. The prophylaxis of transmission of HIV from a mother to the child was conducted. From 28 week-term on she took in azidothymidine. The child was also administered this drug for 7 days after birth. There was no data on PCR testing for HIV. The child was not breast-fed. BCG vaccine was not given. At the age of 2 the child was deleted from the register on the basis of negative serum inspection. The obtained anamnestic data allowed to establish the perinatal infection with HIV.

The following clinical diagnosis was established: HIV infection, IV clinical stage, HIV - associated encephalopathy with a hyperkinetic syndrome, bilateral cerebellum insufficiency, left-side reflex hemiparesis (B23,8). Partial atrophy of visual nerves. Myopia of mild degree of OU. Thrombocytopenia. The persistent generalized lymphadenopathy. Mycotic cheilitis. Mitral valve prolapsed with moderate mitral insufficiency, HO.

This diagnosis was confirmed in the Center for treatment of children with HIV - infection/AIDS at the National children's specialized hospital "OHMATDIT". Highly active antiretroviral therapy (HAART) has been administered to the child: abacavir, lamivudine, efavirenz. On 8th day of treatment abacavir was substituted with tenofovir in view of the allergic reaction. Following the treatment the condition of the child has improved with hyperkinesis amplitude and frequency having diminished considerably.

\section{Discussion}

The lesion of the nervous system in HIV-infected children is one of the most common clinical manifestations of the disease. However, the typical attribute is the development of HIV- encephalop- 
athy in the form of reduced intelligence, impaired concentration and memory. Hyperkinetic syndrome - rare manifestation of nervous system damage in HIV- infected children. Underestimation of the syndrome can cause late diagnosis. To determine the cause of generalized hyperkinesis in the child must be the differential diagnosis of diseases such as acute rheumatic fever, connective tissue diseases, demyelinating diseases, organic pathology of CNS, Wilson - Konovalov disease and HIV - infection.

\section{Conclusion}

The presented clinical supervision demonstrates difficulties in diagnosis of HIV - encephalopathy in a child which was related to a rare clinical presentation with generalized hyperkinesis in the child's age, as well as to the concealment of anamnestic data by the mother regarding her HIV-status.

\section{Bibliography}

1. "HIV infection in Ukraine". Newsletter 50 (2019): 86-88.

2. "Assessment of the regional targeted social program on HIV/ AIDS (analysis period 2009-2016) in Dnipropetrovsk region". Report Kyiv (2018): 12-14.

3. Evtushenko SK and Derevyanko IN. "Neuro-AIDS as one of the urgent problems of modern practical neurology". International Neurological Journal 5.9 (2006): 147-157.

4. Gongora-Rivera F., et al. "The clinical spectrum of neurological manifestations in AIDS patients in Mexico". Archives of Medical Research 31.4 (2000): 393-398.

5. Martynova GP., et al. "A case of severe CNS damage in a child with HIV infection". Childhood Infections 4 (2011): 69-70.

6. Starets 00. "The course, factors of progression and treatment of HIV infection in children”. Odessa (2008): 44.

7. Timchenko VN., et al. "Damage to the nervous system in HIV infection”. Childhood Infections 1 (2009): 32-35.

8. Lowenthal ED., et al. "Neurologic and Psychiatric Manifestations of Pediatric HIV Infection". HIV Curriculum for the Health Professional.

9. Krel R. Central Nervous System Complications in HIV (2011).

10. Donald KA., et al. "Neurologic complications of pediatric human immunodeficiency virus: implications for clinical practice and management challenges in the African setting". Seminars in Pediatric Neurology 21.1 (2014): 3-11.

11. Trillo-Pazos G., et al. "Detection of HIV-1 DNA in microglia/ macrophages, astrocytes and neurons isolated from brain tissue with HIV-1 encephalitis by laser capture microdissection". Brain Pathology 13.2 (2003): 144-154.
12. Manfredi R. "AIDS and Other Manifestations of HIV Infection". Journal of the American Medical Association 293.11 (2005): 1393-1397.

13. Mazus A.I., et al. "Features of the neurological manifestations of HIV infection". Clinical Neurology 3 (2009): 31-32.

14. Khoreva M.A., et al. "Neurological masks of HIV infection". International Research Journal 1.67 (2018): 65-69.

15. Robertson K., et al. "Antiretroviral neurotoxicity". The Journal of Neuro Virology 18.5 (2012): 388-399.

16. Blokhuis C., et al. "Neurodevelopmental delay in pediatric HIV/AIDS: current perspectives". Neurobehavioral HIV Medicine 7 (2016): 1-13.

17. Clinical protocol for the treatment of opportunistic infections and HIV-associated diseases in HIV-infected and AIDS-infected children". Ministry of Health of Ukraine Order № 206 of 07/04 (2006.): 81-82

18. Laughton B., et al. "Early antiretroviral therapy improves neurodevelopmental outcomes in infants". AIDS 26.13 (2012): 1685-1690.

19. Jeremy RJ., et al. "Neuropsychological functioning and viral load in stable antiretroviral therapy-experienced HIV-infected children". Pediatrics 115.2 (2005): 380-387.

20. Shilov GN., et al. "TV MRI aspects of the neuroimaging of CNS lesions in HIV infection". Medical News 12 (2012): 55-57.

21. Seliverstov YuA and Klyushnikov SA. "Differential diagnosis of chorea". Nervous Diseases 1 (2015): 6-15.

22. Piccolo I ., et al. "Cause and course in a series of patient with sporadic chorea”. Journal of Neurology 250 (2003): 429.

23. Sporer B., et al. "HIV-induced chorea: evidence for basal ganglia dysregulation by SPECT". Journal of Neurology 252.3 (2005): 356-358.

\section{Assets from publication with us}

- Prompt Acknowledgement after receiving the article

- Thorough Double blinded peer review

- Rapid Publication

- Issue of Publication Certificate

- High visibility of your Published work

Website: www.actascientific.com/

Submit Article: www.actascientific.com/submission.php Email us: editor@actascientific.com Contact us: +919182824667 